\title{
Analysis of Optical CDMA Signal Transmission: Capacity Limits and Simulation Results
}

\author{
Aminata A. Garba \\ Department of Electrical and Computer Engineering, McGill University, 3480 University Street, Montreal, QC, Canada H3A 2 A7 \\ Email:agarba@tsp.ece.mcgill.ca
}

Raymond M. H. Yim

Division of Engineering and Applied Sciences, Harvard University, Cambridge, MA 02138, USA

Email: ryim@fas.harvard.edu

\begin{abstract}
Jan Bajcsy
Department of Electrical and Computer Engineering, McGill University, 3480 University Street, Montreal, QC, Canada H3A $2 A 7$ Email: jbajcsy@tsp.ece.mcgill.ca

\section{Lawrence R. Chen}

Department of Electrical and Computer Engineering, McGill University, 3480 University Street, Montreal, QC, Canada H3A $2 A 7$ Email: chen@photonics.ece.mcgill.ca
\end{abstract}

Received 5 April 2004; Revised 18 January 2005

\begin{abstract}
We present performance limits of the optical code-division multiple-access (OCDMA) networks. In particular, we evaluate the information-theoretical capacity of the OCDMA transmission when single-user detection (SUD) is used by the receiver. First, we model the OCDMA transmission as a discrete memoryless channel, evaluate its capacity when binary modulation is used in the interference-limited (noiseless) case, and extend this analysis to the case when additive white Gaussian noise (AWGN) is corrupting the received signals. Next, we analyze the benefits of using nonbinary signaling for increasing the throughput of optical CDMA transmission. It turns out that up to a fourfold increase in the network throughput can be achieved with practical numbers of modulation levels in comparison to the traditionally considered binary case. Finally, we present BER simulation results for channel coded binary and $M$-ary OCDMA transmission systems. In particular, we apply turbo codes concatenated with ReedSolomon codes so that up to several hundred concurrent optical CDMA users can be supported at low target bit error rates. We observe that unlike conventional OCDMA systems, turbo-empowered OCDMA can allow overloading (more active users than is the length of the spreading sequences) with good bit error rate system performance.
\end{abstract}

Keywords and phrases: optical CDMA communication, multiple-access channels, $M$-ary modulation, channel capacity, turbo codes.

\section{INTRODUCTION}

Optical CDMA can provide multiple attractive features for data access networks with bursty traffic, that is, flexibility of asynchronous and decentralized network operation, potential of high data throughputs, inherent data security, and potential of total bandwidth utilization by all network users (when utilizing two-dimensional spreading sequences). Ultrahigh transmission rates envisioned for all-optical networks as well as the limited availability of optical domain

This is an open access article distributed under the Creative Commons Attribution License, which permits unrestricted use, distribution, and reproduction in any medium, provided the original work is properly cited. digital logic and signal processing have made OCDMA a challenging research area. Multiple advances and novel techniques have been developed in the past two decades to enable OCDMA technology for access networks $[1,2,3,4,5,6,7$, $8,9,10,11,12,13,14,15,16,17,18]$. Significant research efforts have been made in the design and performance analysis of optical CDMA spreading sequences. The main objective has been to construct new families of optical spreading codes with low cross-correlation values in order to maximize the signal-to-interference ratio and obtain good bit error rate (BER) performances. However, despite the introduction to many families of new OCDMA spreading sequences, severe multiuser interference has usually limited the maximum number of active OCDMA users to between 10 and 20 (at 
target BER of $10^{-9}$ ) and this fact has placed a limit on achieving high aggregate data throughputs in OCDMA networks.

Recently, channel (error-correcting) codes have been considered as a means to reduce the effects of the multiuser interference and improve performance in OCDMA networks $[19,20,21,22]$. It is thus of interest to know the theoretical (Shannon) capacity of an OCDMA network so that the overall system performance can be properly optimized. Channel capacity of optical communication systems has been explored for single-user transmission, for example, in $[23,24]$, taking into account physical limitations of the optical transmission link. Nonetheless, different techniques and approaches have been needed and utilized so far to analyze OCDMA capacity limitations in the presence of multiple users. Authors in references $[25,26]$ explored fundamental limitations under specific OCDMA architectures, for example, on-off keying, specific optical receivers, and code correlation constraints in [25]. Hence, the motivation of this paper is first to determine capacity limits on binary OCDMA transmission assuming that the best possible spreading sequences, single-user receiver, and channel codes are utilized. Consequently, we study the effects of using more than two modulation levels on increasing the capacity of OCDMA transmission with single-user detection at the receiver. Finally, we use powerful channel codes, such as turbo codes concatenated with Reed-Solomon codes, to achieve up to several hundred users in OCDMA systems with a target BER of $10^{-9}$.

This paper is organized as follows. Section 2 presents channel models for binary and $M$-ary OCDMA transmission when single-user detection is applied at the receiver and considers the noiseless, interference-limited transmission. Section 3 calculates the channel capacity limits on OCDMA network throughput under these assumptions and analyzes the benefit of using $M$-ary OCDMA modulation to improve upon the capacity of the binary systems. Furthermore, for high modulation levels, we propose a specific input distribution that satisfactorily increases the throughput of the $M$-ary OCDMA compared to the equiprobable signaling. In Section 4, we evaluate the impact of Gaussian (photodetector) noise on the throughput limits derived previously. Section 5 proposes and explores coded OCDMA architectures utilizing turbo codes and these are shown to significantly improve the overall system performance in OCDMA networks. Finally, concluding remarks and directions of further work are given in Section 6.

\section{CHANNEL MODELS}

\subsection{General assumptions}

We consider the general case of $M$-ary OCDMA transmission $(M \geq 2)$ with bit-asynchronous, chip-synchronous transmission when $K$ users are sending information simultaneously through the network using a laser source that can be intensity modulated to one of $M$ modulation levels $(0,1, \ldots, M-1)$. Each user's data are spread and encoded prior to being sent over the shared channel and the users behave in an independent (i.e., no collaboration is allowed)

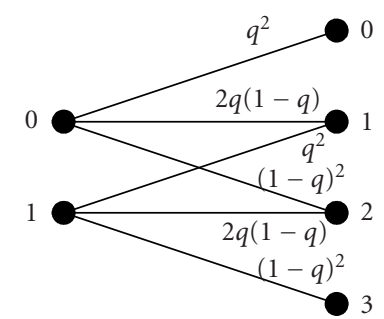

FIGURE 1: Chip-level discrete memoryless channel model of a 3-user asynchronous OCDMA system with single-user detection. Please note that the channel input symbols (chips) are used with probabilities $p_{0}=q$ and $p_{1}=1-q$.

and symmetric manner (i.e., they all have the same probability of using a given modulated symbol in their spreading sequence). We will also assume that the receiver performs single-user detection (SUD), where we can assume without any loss of generality that the first user is the decoded user. Our capacity analysis is carried out on the chip level in a general sense, assuming that the best possible singleuser detector, spreading and error-control coding are used. In Section 5, we will deal with specific architectures and explore their performances through simulations results.

We will start our analysis with the interference-limited (noiseless) transmission case, that is, the effects of receiver and channel noise are assumed negligible when compared to the interference of other users who are also transmitting their data over the shared multi-access channel. Under these assumptions, the OCDMA transmission can be modeled using an appropriate discrete memoryless channel.

\subsection{Examples of channel models for OCDMA transmission with SUD}

Consider the case of 3 users transmitting binary OCDMA chip symbols 1 and 0 over the optical channel with probabilities $p_{0}=q$ and $p_{1}=1-q$, respectively. We can model such transmission using a discrete memoryless channel (DMC) shown in Figure 1. At the output of the channel, the input signal of user 1 is corrupted by interference due to other simultaneous users of the OCDMA network. For this 3-user binary network, if user 1 transmits chip symbol 0 , the receiver will receive 0 if and only if the other 2 users also transmit zero. Hence the transition probability is $q^{2}$. Similarly, the receiver takes the value 1 if one interfering user transmits the chip symbol 1 and the other transmits 0 , which occurs with probability $2 q(1-q)$.

As a second example, we consider the case of 2 OCDMA users with ternary modulation, with corresponding DMC representation shown in Figure 2. At the chip level, each user can send symbols 0,1 , or 2 with probabilities $p_{0}, p_{1}$, and $p_{2}=\left(1-p_{0}-p_{1}\right)$, and the channel transition matrix is hence given by

$$
P_{Y \mid X_{1}}=\left(\begin{array}{ccccc}
p_{0} & p_{1} & p_{2} & 0 & 0 \\
0 & p_{0} & p_{1} & p_{2} & 0 \\
0 & 0 & p_{0} & p_{1} & p_{2}
\end{array}\right) .
$$




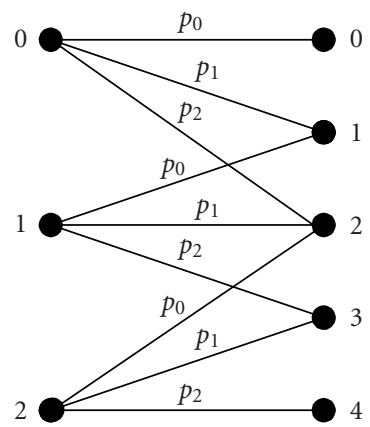

FIgURe 2: Chip-level DMC model for a 2-user ternary $(M=3)$ OCDMA transmission with single-user detection. Channel input symbols are used with probabilities $p_{0}, p_{1}$, and $p_{2}=\left(1-p_{0}-p_{1}\right)$.

The channel output $Y$ is the real sum of the symbol intensities sent by all simultaneous users and can hence take values $0,1,2,3$, or 4 with probabilities $p_{0}^{2}, 2 p_{0} p_{1}, p_{1}^{2}+2 p_{0} p_{1}, 2 p_{1} p_{2}$, and $p_{2}^{2}$ respectively. If we evaluate the information-theoretic capacity of this specific channel, we find out that such a system is limited to throughput of 0.74 bits per OCDMA chip for the decoded user and thus the cumulative throughput for the 2 independent users is limited to 1.48 bits per OCDMA chip.

It is also interesting to note from these examples that the channel matrix depends on the input probability distribution. Consequently, the DMC representing the OCDMA system is not a constant (fixed) channel like the majority of channels considered in information theory [27]. As we will see in Section 4, this variability of the channel will lead to a difficult optimization problem of enumerating the numerical value of the capacity.

\subsection{M-ary OCDMA transmission model}

More generally, at the chip level, the DMC representation of a $K$-user $M$-ary modulated OCDMA transmission has as input the random variable $X_{1}$ corresponding to the information sent by the desired user and taking value on the alphabet $\mathbf{X}=\{0,1, \ldots, M-1\}$; and as an output the real sum

$$
Y=\sum_{i=1}^{K} X_{i}
$$

where $X_{i}$ for $i=1,2, \ldots, K$ is a sequence of independent identically distributed random variables corresponding to chip symbols transmitted by users $1,2, \ldots, K$ after OCDMA spreading and error-control coding. Therefore, the output alphabet is given by $\mathbf{y}=\{0,1,2, \ldots, K \times(M-1)\}$.

The entries of the channel matrix $P_{Y \mid X_{1}}$ are the conditional probabilities of the output symbol, given the input symbol and can be described for all $y \in \mathbf{y}$ and $x_{1} \in \mathbf{X}$ as follows:

$$
P_{Y \mid X_{1}}\left(Y=y \mid X_{1}=x_{1}\right)=\sum_{\begin{array}{c}
\left(x_{2}, x_{3}, \ldots, x_{K}\right) \in \mathbf{X}^{K-1} \\
\text { s.t. } \\
x_{2}+x_{3}+\cdots+x_{K-1}=\left(y-x_{1}\right)
\end{array}} p_{x_{2}} p_{x_{3}} \cdots p_{x_{K}} .
$$

Consequently, it can be again noticed that the channel depends on the input probability mass function for a general $K$ user, $M$-ary OCDMA transmission with SUD. Furthermore, the output probability distribution is given by the following expression for all $y \in \mathbf{y}$ :

$$
P_{Y}(Y=y)=\sum_{\substack{\left(x_{1}, x_{2}, x_{3}, \ldots, x_{K}\right) \in \chi^{K} \\ \text { s.t. } \\ x_{1}+x_{2}+x_{3}+\cdots+x_{K}=y}} p_{x_{1}} p_{x_{2}} \cdots p_{x_{K}}
$$

The values of the entries of the channel transition matrix $P_{Y \mid X_{1}}\left(Y=y \mid X_{1}=x_{1}\right)$ as well as the output probabilities $P_{Y}(Y=y)$ can be found using generating functions. For $K$ users sending data over an optical $M$-ary modulated channel, the conditional probabilities assuming that the input symbol is $x_{1} \in \mathbf{X}$ are generated by the following polynomial in the symbolic variable $z$ :

$$
R(z)=z^{x_{1}}\left(p_{0} z^{0}+p_{1} z^{1}+\cdots+p_{M-1} z^{M-1}\right)^{K-1} .
$$

To obtain the resulting probabilities, we expand this polynomial in the form

$$
R(z)=\alpha_{x_{1}} z^{x_{1}}+\alpha_{x_{1}+1} z^{x_{1}+1}+\cdots+\alpha_{x_{1}+(K-1)(M-1)} z^{x_{1}+(K-1)(M-1)}
$$

and the conditional probabilities on the channel are given by $P_{Y \mid X_{1}}\left(Y=y \mid X_{1}=x_{1}\right)=\alpha_{y+x_{1}}$ for all $y \in \mathbf{y}=$ $\{0,1, \ldots, K \times(M-1)\}$ and $x_{1} \in \mathbf{X}$. Similarly, the generating polynomial in the symbolic variable $z$ for the output distribution is given by

$$
Q(z)=\left(p_{0} z^{0}+p_{1} z^{1}+\cdots+p_{M-1} z^{M-1}\right)^{K} .
$$

To obtain the output probabilities, we expand the previous polynomial in the form

$$
Q(z)=\alpha_{0} z^{0}+\alpha_{1} z^{1}+\cdots+\alpha_{K(M-1)} z^{K(M-1)}
$$

and the output probabilities are given by $P_{Y}(Y=y)=\alpha_{y}$ for $y \in \mathbf{y}=\{0,1, \ldots, K \times(M-1)\}$.

If we consider, for instance, the case of binary modulation $(M=2)$ with input alphabet $\mathbf{X}=\{0,1\}$ and the output alphabet $\mathbf{y}=\{0,1,2, \ldots, K\}$, the channel conditional probabilities are given by

$$
P_{Y \mid X_{1}}\left(Y=y \mid X_{1}=x_{1}\right)=\left(\begin{array}{l}
K-1 \\
y-x_{1}
\end{array}\right)(1-q)^{y-x_{1}} q^{K-1-y+x_{1}},
$$

where $p_{0}=q, p_{1}=1-q$ and, for notational convenience, we define $\left(\begin{array}{c}K-1 \\ -1\end{array}\right)=\left(\begin{array}{c}K-1 \\ K\end{array}\right)=0$. Due to the symmetry of the problem, the channel matrix of conditional probabilities can be written as

$$
P_{Y \mid X_{1}}=\left(\begin{array}{ccccc}
\beta_{0} & \beta_{1} & \cdots & \beta_{K-1} & 0 \\
0 & \beta_{0} & \beta_{0} & \cdots & \beta_{K-1}
\end{array}\right),
$$




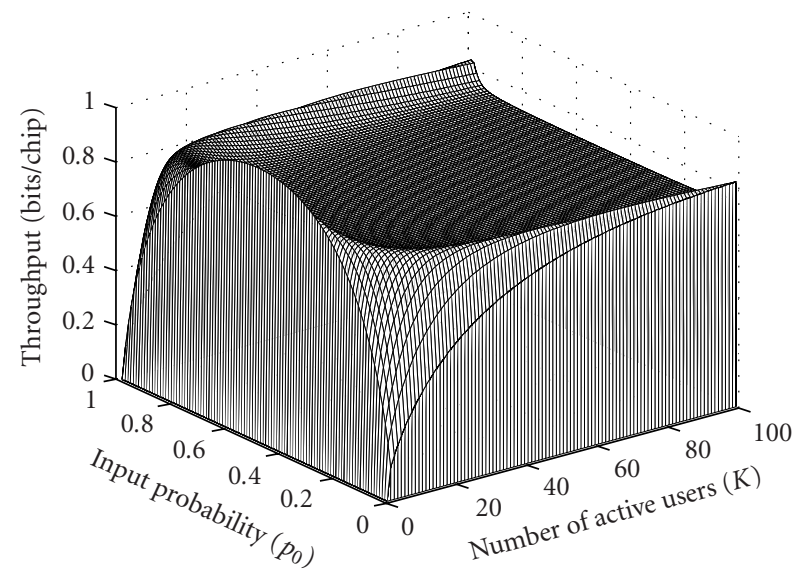

FIgURE 3: Fundamental limits on asynchronous binary OCDMA transmission.

where $\beta_{i}=\left({ }^{K-1}\right)(1-q)^{i} q^{K-1-i}$. The channel output probability is consequently given by

$$
P_{Y}(Y=y)=\left(\begin{array}{c}
K \\
y
\end{array}\right)(1-q)^{y} q^{K-y} .
$$

\section{CAPACITY EVALUATION}

\subsection{Information capacity of the binary OCDMA transmission with SUD}

The information-theoretical (Shannon) capacity of the binary optical CDMA transmission with single-user detection at the receiver can be calculated using the channel model described in (9). Due to the symmetrical and independent operation of the $K$ users on the OCDMA channel, the cumulative throughput by all the $K$ simultaneous users on the channel is limited in (information) bits per transmitted OCDMA chip by

$$
C_{2, K}=K \sup _{q \in[0,1]} I_{2, K}\left(X_{1}, Y\right)
$$

where $I_{2, K}\left(X_{1}, Y\right)$ is given by (13) below and represents overall mutual information of a $K$-user binary OCDMA transmission with single-user detection.

The aggregate mutual information of such a bitasynchronous binary OCDMA channel is plotted as a function of the input probability $q=P\left(X_{1}=0\right)$ in Figure 3 for various numbers of users. When only 1 user is active in the system, without the presence of noise, the channel reduces to a perfect noiseless binary channel and the capacity is 1 bit per chip. As the number of users increases, the interference reduces the theoretical capacity limit until it reaches a steady-state value. For example, for a 50 -user transmission, the aggregate capacity is 0.8374 bits per chip achieved when the input probability is $q=0.03$. Note that the uniform distribution does not achieve the capacity and for the same system, it yields to a throughput limit of around 0.7288 bits per

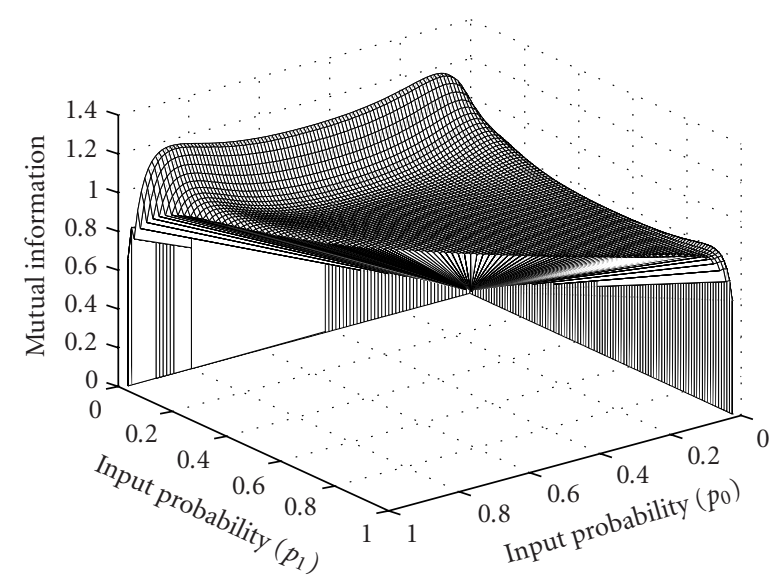

FIgUre 4: Aggregate mutual information as a function of input probability for 10 users, ternary OCDMA transmission.

\section{OCDMA chip.}

$$
\begin{aligned}
I_{2, K}\left(X_{1} ; Y\right) & \\
= & -\sum_{i=0}^{K}\left(\begin{array}{c}
K \\
i
\end{array}\right)(1-q)^{i} q^{K-i} \log _{2}\left(\left(\begin{array}{c}
K \\
i
\end{array}\right)(1-q)^{i} q^{K-i}\right) \\
& +\sum_{i=0}^{K-1}\left(\begin{array}{c}
K-1 \\
i
\end{array}\right)(1-q)^{i} q^{K-1-i} \\
& \times \log _{2}\left(\left(\begin{array}{c}
K-1 \\
i
\end{array}\right)(1-q)^{i} q^{K-1-i}\right) .
\end{aligned}
$$

\subsection{Information capacity with nonbinary modulation}

Due to the symmetrical and independent operation of the $K$ users on the channel, the cumulative throughput for all the $K$ users is limited by

$$
C_{M, K}=K \sup _{P_{X_{1}}} I_{M, K}\left(X_{1} ; Y\right) .
$$

Moreover, due to the symmetry of the channel matrix $P_{Y \mid X_{1}}$ in (3) (rows are cyclical shift of each other), it follows that $H\left(Y=y \mid X_{1}=x_{1}\right)=H\left(Y=y \mid X_{1}=0\right)$ for all $x_{1}=1,2, \ldots, M-1$. Consequently, the mutual information $I_{M, K}(X ; Y)$ for a $K$-user $M$-ary OCDMA transmission with single-user detection can be expressed as in (15).

Generally, the variability of the discrete memoryless channel modeling the $M$-ary OCDMA transmission on the input probability mass function causes a non-concavity of the mutual information $I_{M, K}\left(X_{1} ; Y\right)$. For example, Figure 4 shows the overall mutual information as a function of the input probability distribution and its non-concave nature in the case of ternary $(M=3)$ modulation and transmission by $K=10$ active users. This distinguishes the $M$-ary multiaccess channel with single-user detection from the standard class of fixed channels where the mutual information is concave in the channel input distribution [27], and hence finding this capacity requires global optimization tools. 


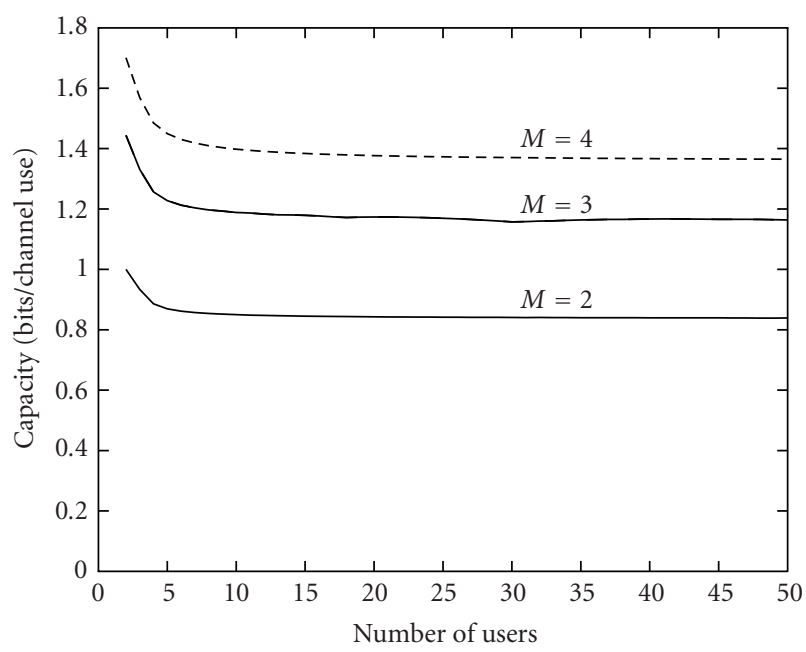

FIGURE 5: Aggregate capacity of OCDMA transmission as a function of the number of users $K$ shown for $M=2,3$, and 4 modulation levels.

Indeed, the commonly used numerical algorithms for evaluating the channel capacity (e.g., the Arimoto-Blahut algorithm [28]) do not apply in this case, since they generally assume and utilize concavity of the mutual information in the channel input distribution.

$$
\begin{aligned}
& I_{M, K}\left(X_{1} ; Y\right) \\
& =\sum_{y=0}^{(K-1)(M-1)}\left(\sum_{\substack{\left(x_{2}, x_{3}, \ldots, x_{K}\right) \in \chi^{K-1} \\
\text { s.t. } \\
x_{2}+x_{3}+\cdots+x_{K}=y}} p_{x_{2}} p_{x_{3}} \cdots p_{x_{K}}\right) \\
& \times \log _{2}\left(\sum_{\substack{\left(x_{2}, x_{3}, \ldots, x_{K}\right) \in \chi^{K-1} \\
\text { s.t. } \\
x_{2}+x_{3}+\cdots+x_{K}=y}} p_{x_{2}} p_{x_{3}} \cdots p_{x_{K}}\right) \\
& -\sum_{y=0}^{K(M-1)}\left(\sum_{\substack{\left(x_{1}, x_{2}, x_{3}, \ldots, x_{K}\right) \in \mathbf{X}^{K} \\
x_{1}+x_{2}+x_{3}+\ldots+x_{K}=y}} p_{x_{1}} p_{x_{2}} \cdots p_{x_{K}}\right) \\
& \times \log _{2}\left(\sum_{\substack{\left(x_{1}, x_{2}, x_{3}, \ldots, x_{K}\right) \in \mathbf{X}^{K} \\
x_{1}+x_{2}+x_{3}+\cdots+x_{K}=y}} p_{x_{1}} p_{x_{2}} \cdots p_{x_{K}}\right) \text {. }
\end{aligned}
$$

Due to the difficulty of having an exact analytical expression for the capacity of the OCDMA transmission with single-user detection, we numerically compute the capacity of the channel and these results are shown in Figure 5 for selected numbers of modulation levels. It can then be seen that the throughput of this system is maximum for a 2-user system and reaches a limiting value for increasing number of

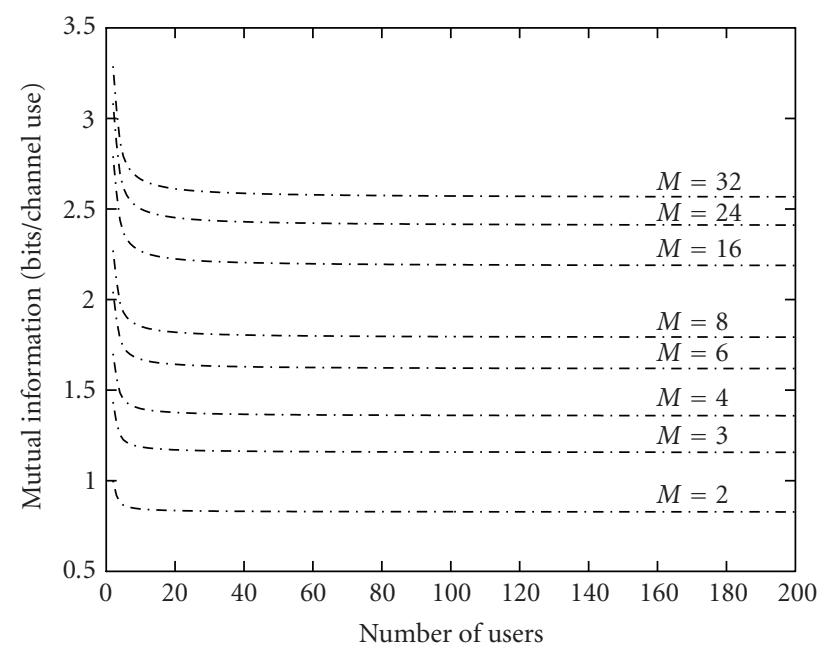

FIGURE 6: Mutual information using the proposed input probability distribution.

users. Consequently, when the number of users is sufficiently large $(K \geq 10)$, the aggregate throughput of the OCDMA transmission is stable, quite independent of the total number of users. This is a highly desirable feature in a network with bursty traffic, since congestion does not occur as the number of users increases.

It is interesting to compare the capacity with the throughput achieved for uniformly distributed inputs. In this case, the aggregate mutual information for large enough number of users is much lower than the capacity of the channel shown in Figure 5 and is approximately

$$
K I_{M, K}\left(X_{1} ; Y\right) \approx K \log _{2}\left(\sqrt{\frac{K}{K-1}}\right) \longrightarrow 0.72 \text { bits/chip. }
$$

\subsection{Near-capacity-achieving input distributions}

Although the numerical evaluation gives a way of determining the capacity of an $M$-ary OCDMA network, this process can still be difficult and computational intensive, especially for many modulation levels. This is explained by the high dimensionality of the problem added to the difficulty of global optimization needed to maximize the mutual information. Hence, to allow less complex, approximate evaluation, we heuristically propose the following class of input probability distributions that satisfactorily increases the throughput of the system:

$$
\begin{gathered}
p_{0}=p_{M-1}=\frac{K-1}{2 K} \\
p_{1}=p_{2}=\cdots=p_{M-2}=\frac{1}{K(M-2)} .
\end{gathered}
$$

The system throughput can then be viewed as the binary channel information due to the two most used symbols, 


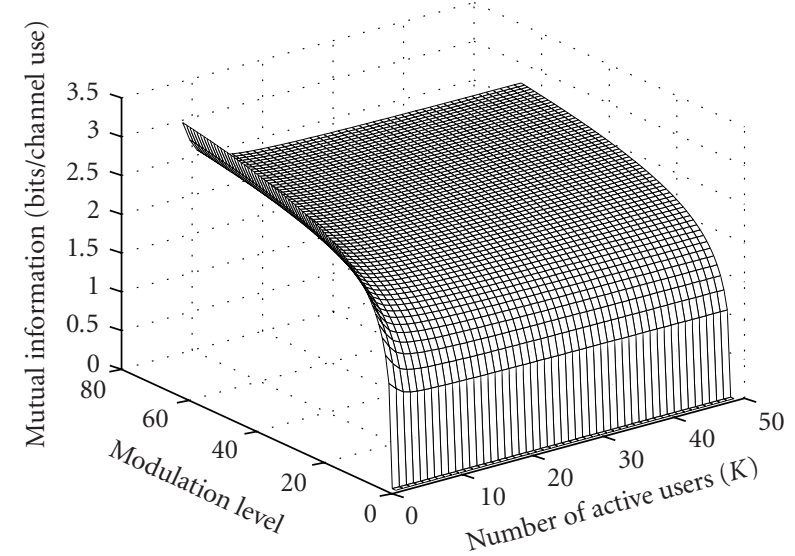

FIGURE 7: Mutual information as a function of the modulation levels and the number of users.

0 and $M-1$, plus additional information corresponding to the contribution of the $M-2$ unlikely symbols $1,2, \ldots, M-2$.

The mutual information, obtained using this proposed input probability distribution, is plotted as a function of the number of active users in Figure 6 and as a joint function of the number of users and the $M$-ary modulation levels in Figure 7. For $M=2,3$, and 4 modulation levels, this mutual information is approximately equal to the actual capacity of the system (shown originally in Figure 5). As an example, the mutual information using this distribution for a 25user ternary communication system is about 1.159 , identical within the first 4 digits to the capacity of the system found by global optimization.

It is interesting to note that the throughput increases approximately logarithmically with the increasing number of modulation levels $M$ as $K I_{M, K}(X, Y) \approx 0.36 \log _{2}(M-1)+$ 0.83 bits per chip for $M>2$ and $K>10$. It may be possible to increase the throughput of the OCDMA by increasing the $M$-ary modulation level as desired. However, as the number of the modulation levels $M$ increases, the information gained becomes less important. Therefore, an OCDMA system may be implemented with a suitable choice of $M$, creating a compromise between complexity and throughput.

\section{IMPACT OF NOISE ON THE THROUGHPUT OF OCDMA TRANSMISSION WITH SUD}

So far, we have omitted the presence of channel noise. Although this gives an upper bound on the capacity of OCDMA transmission under perfect conditions, noise should be included in the analysis to assess the robustness of such results. We will assume that the channel is corrupted by an additive white Gaussian noise (AWGN) which models the electronic (photodetector) noise which may degrade the sent information [11]. The input of the channel is still the random variable $X_{1}$ taking values on the alphabet $\mathbf{X}=$ $\{0,1, \ldots, M-1\}$ with the probabilities derived in the previous section. However, the channel output is now the continuous random variable $Z=Y+N$, where $Y$ is the output of

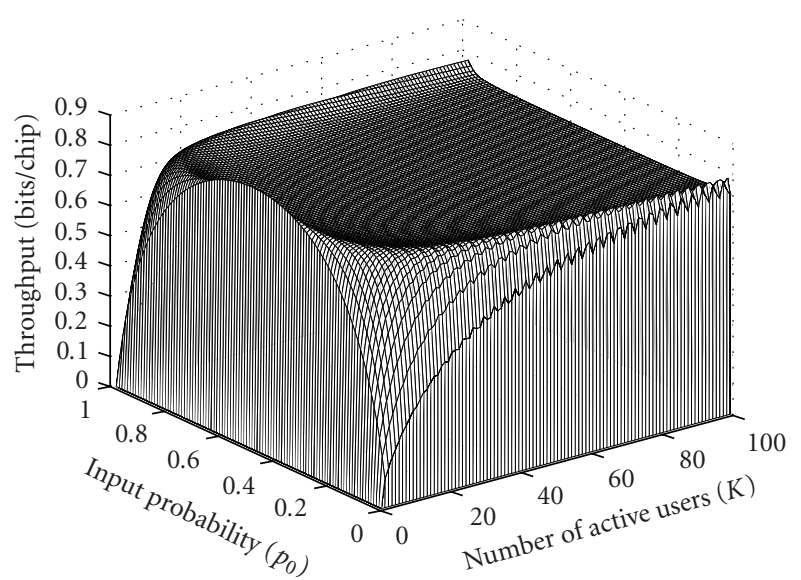

(a)

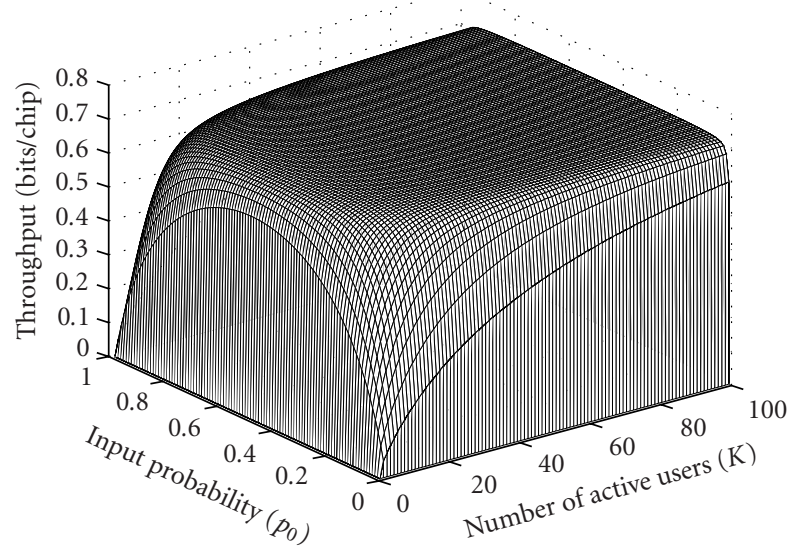

(b)

FIGURE 8: Throughput limit on binary asynchronous OCDMA transmission when AWGN is present for noise variance: (a) $\sigma^{2}=$ 0.09 and (b) $\sigma^{2}=0.25$.

the noiseless channel from (2) and $N$ is a Gaussian random variable with zero mean and variance of $\sigma^{2}$.

\subsection{Impact of noise on the binary OCDMA transmission}

The aggregate Shannon capacity of the binary OCDMA channel with SUD has the form of (12), but the mutual information term is modified to $I_{2, K}^{\mathrm{AWGN}}\left(X_{1} ; Z\right)$ due to the additive white Gaussian noise (AWGN) corrupting the received data and is described by (18) below. This new mutual information is plotted as a function of the input distribution and the number of active users in Figure 8 for two different values of noise variance. In Figure 9, the overall throughput limit for a 20-user binary OCDMA transmission is shown as a function of the input probability for selected noise variances. Note that for high enough signal-to-noise ratios, the result approaches the interference-limited scenario, however for extremely low SNR (for noise variance of 0.25 , the SNR is 


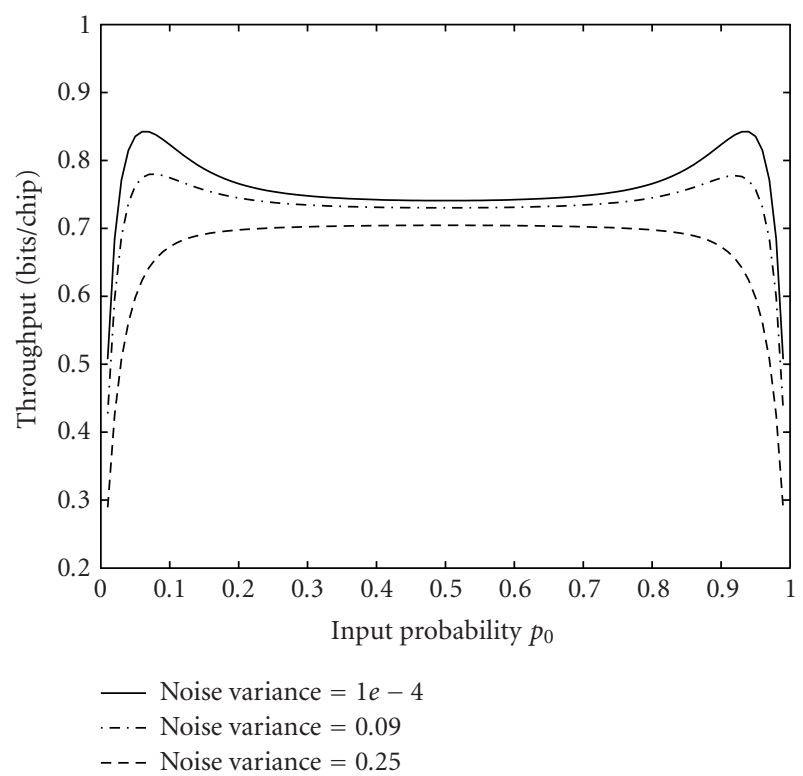

FIGURE 9: Limit on aggregate throughput of a 20-user binary OCDMA system at different noise variances with different input distribution.

approaching zero $\mathrm{dB}$ ), the throughput starts decreasing:

$$
\begin{aligned}
& i_{2, K}^{\mathrm{AWGN}}\left(X_{1} ; Z\right) \\
& =-\int_{-\infty}^{\infty}\left(\sum_{j=0}^{K}\left(\begin{array}{c}
K \\
j
\end{array}\right)(1-q)^{j} q^{K-j} \frac{1}{\sqrt{2 \pi \sigma^{2}}} \exp \left(-\frac{(z-j)^{2}}{2 \sigma^{2}}\right)\right) \\
& \times \log _{2}\left(\sum_{j=0}^{K}\left(\begin{array}{c}
K \\
j
\end{array}\right)(1-q)^{j} q^{K-j} \frac{1}{\sqrt{2 \pi \sigma^{2}}}\right. \\
& \left.\times \exp \left(-\frac{(z-j)^{2}}{2 \sigma^{2}}\right)\right) d z \\
& +\int_{-\infty}^{\infty}\left(\sum_{i=0}^{K-1}\left(\begin{array}{c}
K-1 \\
i
\end{array}\right)(1-q)^{i} q^{K-1-i} \frac{1}{\sqrt{2 \pi \sigma^{2}}}\right. \\
& \left.\times \exp \left(-\frac{(z-i)^{2}}{2 \sigma^{2}}\right)\right) \\
& \times \log _{2}\left(\sum_{i=0}^{K-1}\left(\begin{array}{c}
K-1 \\
i
\end{array}\right)(1-q)^{i} q^{K-1-i} \frac{1}{\sqrt{2 \pi \sigma^{2}}}\right. \\
& \left.\times \exp \left(-\frac{(z-i)^{2}}{2 \sigma^{2}}\right)\right) d z
\end{aligned}
$$

\subsection{Impact of noise on the M-ary OCDMA transmission}

To explore the noise resistance/sensitivity of the results computed so far, we compute the throughput limits on the OCDMA transmission in the presence of AWGN. We will use the noiseless capacity-achieving distribution in case of $M=2,3,4$ modulation levels and the proposed nearcapacity-achieving input distributions from Section 3.3 for

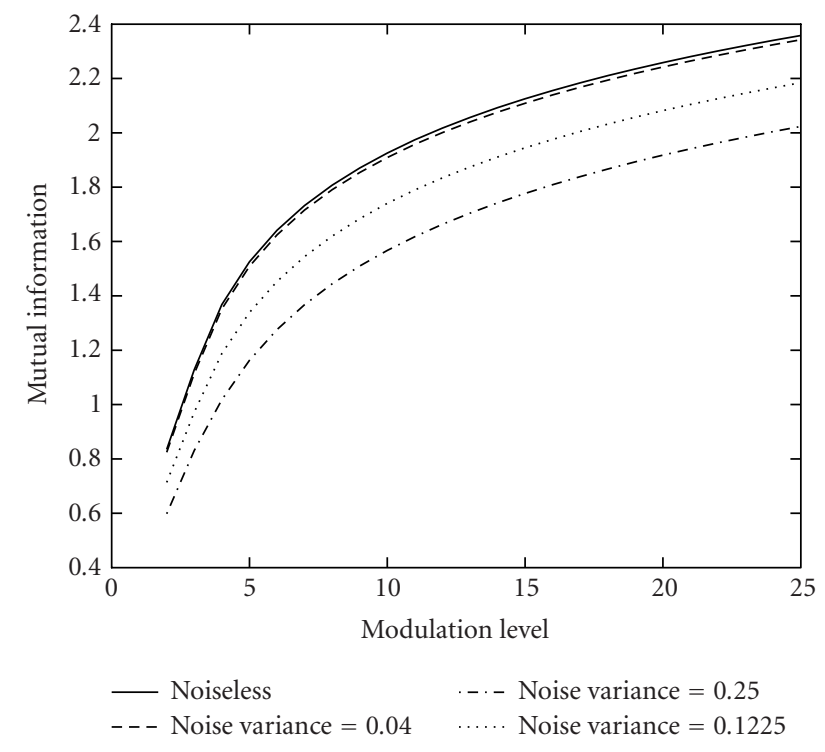

FIGURE 10: Mutual information as a function of the number of modulation levels $M$ for a 20-user system.

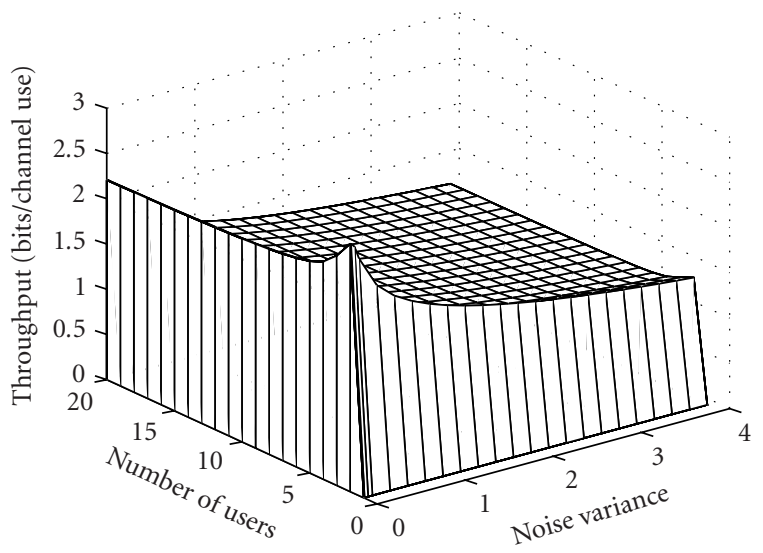

FIGURE 11: Mutual information as a function of the number of users and the noise variance for a 16-ary OCDMA transmission.

$M>4$ modulation levels. The aggregate throughput of the OCDMA transmission under these assumptions is limited by $K I_{M, K}^{\mathrm{AWGN}}\left(X_{1} ; Z\right)$, where the mutual information $I_{M, K}^{\mathrm{AWGN}}\left(X_{1} ; Z\right)$ is given by (18).

Figure 10 shows the aggregate throughput limits on a 20user OCDMA network for selected noise variances, plotted as a function of the modulation levels number. For small noise variances, the noisy throughput limits almost overlap with the noiseless system throughput limits.

The throughput limits are reduced for increasing noise power, but even in this case, the throughput increase with nonbinary modulation is satisfactory. In Figure 11, we show the aggregate throughput limit against the noise variance and the number of users for 16-ary OCDMA modulation. It can be seen that even for high noise variance, the transmission is still reasonably robust to noise, since the throughput limit does not experience a sharp drop due to the noise. 


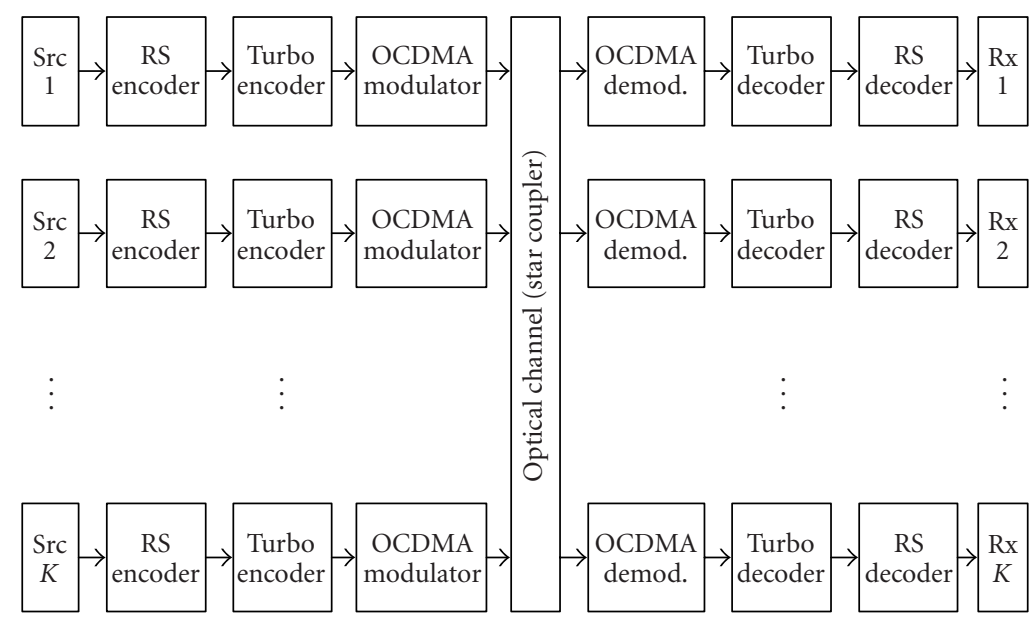

FIGURE 12: Schematic block diagram of the proposed OCDMA transmission scheme for binary and nonbinary modulation.

\section{CODED OCDMA TRANSMISSION WITH TURBO AND REED-SOLOMON CODES}

Multi-user interference usually severely limits performance of OCDMA systems without error-control coding. Systems based on optical spreading sequences only can usually support between 10 to 20 simultaneously transmitting users with bit error rates below $10^{-9}$ (standard performance benchmark for OCDMA transmission). Alternatively, one can compare OCDMA systems using normalized spectral efficiency, that is, aggregate network throughput measured in information bits per OCDMA chip. Traditional (uncoded) OCDMA systems achieve aggregate system throughputs of about 0.03 bits per OCDMA chip, much below the predicted capacity limits. Consequently, we propose several coded OCDMA transmission schemes to increase the OCDMA throughput.

\subsection{Coded binary OCDMA systems}

In the proposed transmission scheme in Figure 12 data of each user are first encoded by a high-rate Reed-Solomon code and interleaved (permuted), then encoded by a turbo code and finally modulated by a user-specific binary optical spreading sequence, such as two-dimensional wavelengthtime balanced codes for differential detection (BCDD) [17]. The role of the optical spreading sequence is to match the data to the channel and to provide low cross-correlation between data sent by different users. The role of the turbo code is to achieve an intermediate bit error rate of $10^{-3}$ to $10^{-5}$, while the Reed-Solomon code provides final error rate at the receiver below the desired error rate of $10^{-9}$. At the receiver end, optical matched filtering and consequent sampling are performed using the optical spreading sequence of the desired user. Turbo decoding followed by Reed-Solomon decoding are used to recover data from the effects of multi-user interference and channel noise.

We first present BER performance of the proposed binary OCDMA system when each user employs a $(255,239)$ ReedSolomon code over $G F(256)$ [29], a rate $1 / 2$ (or 1/3) turbo code and a $\operatorname{BCDD}(32,16,0.156)$ wavelength-time optical spreading sequence (please see [17] for notation). The turbo code is based on the original rate 1/3 turbo coding scheme [30] which uses a 16-state recursive systematic code with generator polynomial $(37,21)$. The rate of $1 / 2$ is obtained through puncturing; a packet of length of 10000 bits is used with an s-rand interleaver of spread 40. Consequent application of the Reed-Solomon decoder decreases the overall BER below $10^{-9}$, as shown in Figure 13b. In terms of channel efficiency, this corresponds to an aggregate system throughput of 0.37 information bits per OCDMA chip.

Figure 14 shows the BER performance when the rate $1 / 3$ turbo code is utilized. Simulation results show that up to 700 users can be supported with a BER below $10^{-9}$ after 8 turbo decoding iterations and RS decoding are performed. This corresponds to an aggregate throughput of 0.42 bits per OCDMA chip. In terms of transmission rate over the optical channel, if each user transmits using 32 wavelengths at OC-12 chip rate and 16-time chips per bit, the proposed scheme would achieve an effective aggregate throughput of $8.4 \mathrm{Gbps}=(700 \times(1 / 3) \times(239 / 255) \times(1 / 16)$ $\times 620$ Mbps).

Finally, Figure 15 shows the BER performance for the system when noise is present with $\sigma^{2}=0.09$. With rate $1 / 3$ turbo code, spreading length of 512, more than 650 users can be supported, which corresponds to a throughput of 0.40 bits per OCDMA chip.

\subsection{Coded M-ary OCDMA transmission}

We considered the coded $M$-ary OCDMA transmission scheme shown in Figure 12. Each user's data are first encoded by a $(255,239)$ Reed-Solomon code over $G F(256)$ followed by a rate $1 / 3$ turbo code, then modulated using a user-specific $M$-ary optical spreading sequence corresponding to bits 0 and 1 . The $M$-ary OCDMA spreading sequences are onedimensional codes composed of symbols $m=0,1, \ldots, M-$ 1 pseudorandomly generated with probabilities chosen according to the capacity-achieving input probability distribution of (17). For example, for ternary OCDMA transmission with 16 aggregate users, the spreading codes are generated 


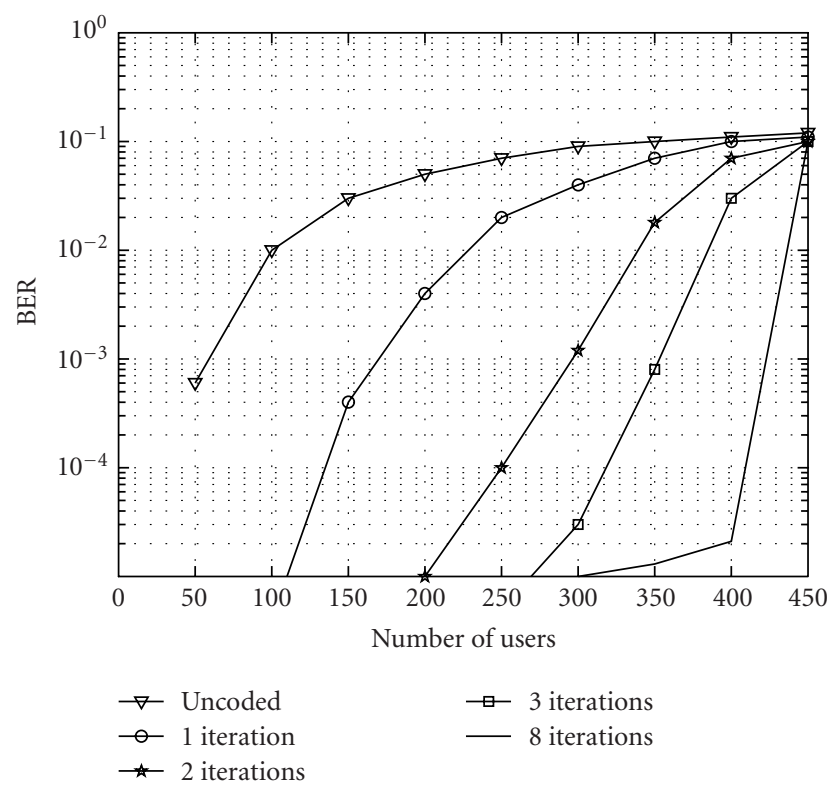

(a)

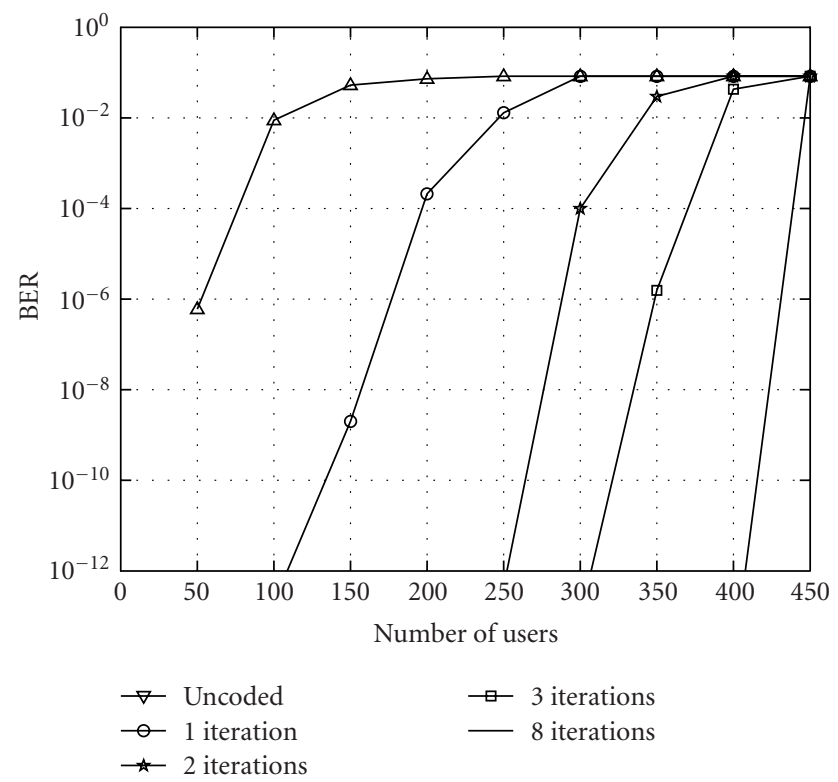

(b)

FIGURE 13: BER performance of the proposed coded OCDMA system as a function of the number of active users: (a) after rate 1/2 turbo decoding; (b) after turbo and RS decoding.

using symbols 0,1 , and 2 with probability distribution corresponding to $p_{1} \approx 1 / 16$ and $p_{0} \approx p_{2} \approx 15 / 32$. The OCDMA modulator serves as an interface between the user's data and the optical channel and the purpose of using such spreading sequences is to aim at achieving the capacity from the theoretical model of the previous sections. The optical channel is modeled by a $K$-user adder channel affected by AWGN

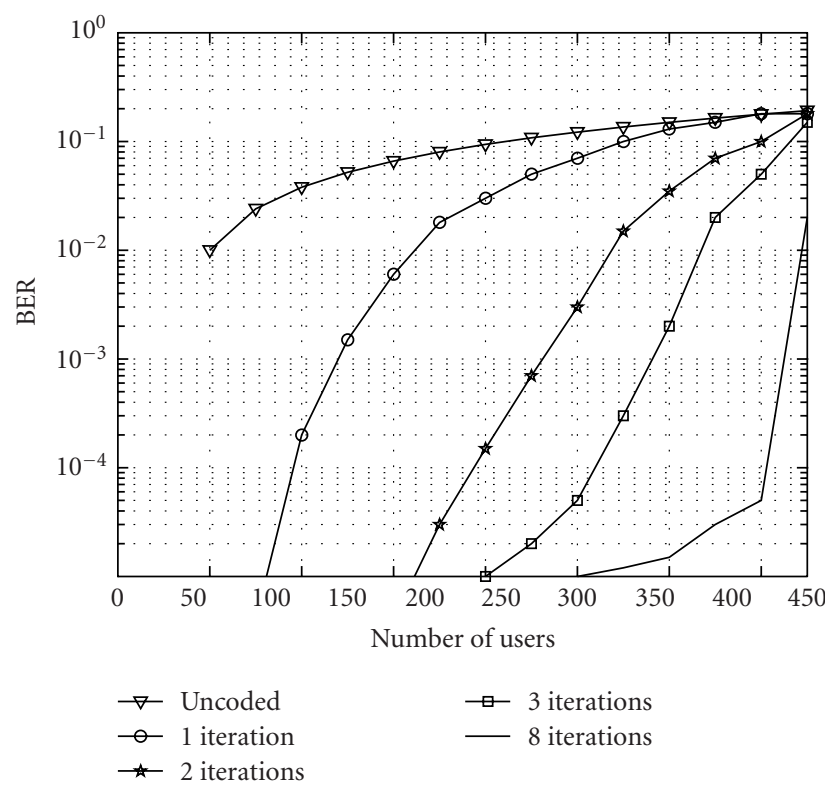

(a)

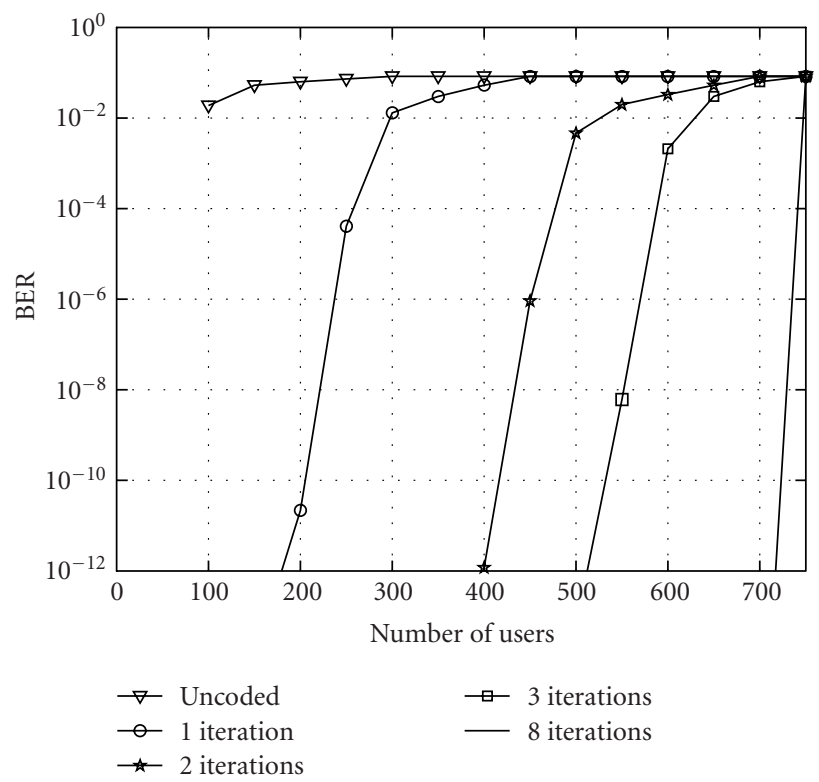

(b)

FIGURE 14: BER performance of the proposed coded OCDMA system as a function of the number of active users: (a) after rate $1 / 3$ turbo decoding; (b) after turbo and RS decoding.

channel noise. Given the channel observations on the chip level, the $M$-ary OCDMA demodulator uses an appropriate channel model described in Section 2 to estimate the a posteriori probabilities of chip-symbol from set $\{0,1,2$, $M-1\}$ being transmitted by the desired user. Consequently, these estimates are combined using the $M$-ary optical spreading sequence of this user via multiplying the appropriate 


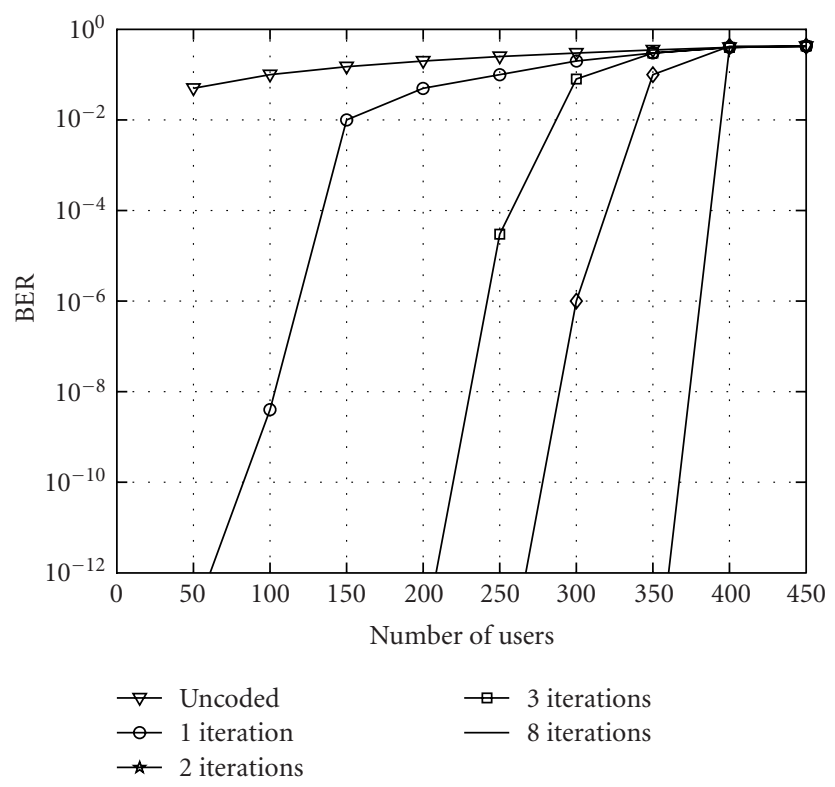

(a)

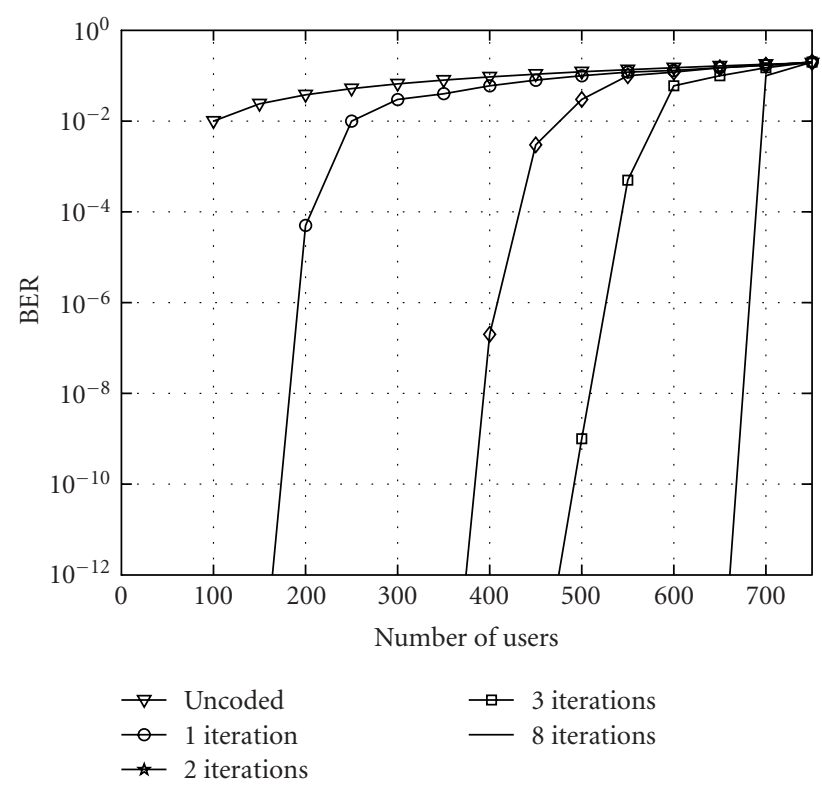

(b)

FIGURE 15: BER performance of the proposed coded OCDMA system in the presence of noise $\left(\sigma^{2}=0.09\right)$ as a function of the number of active users: (a) after rate $1 / 2$ turbo and RS decoding; (b) after rate $1 / 3$ turbo and $\mathrm{RS}$ decoding.

probabilities, thus estimating the probability of transmitted coded bits. The performance of the system is measured in terms of the bit error rate of a desired user.

We simulated the bit error rates of selected turbo- and RS-coded $M$-ary OCDMA systems for the transmission model described in Figure 12 and for optical signal-to-noise

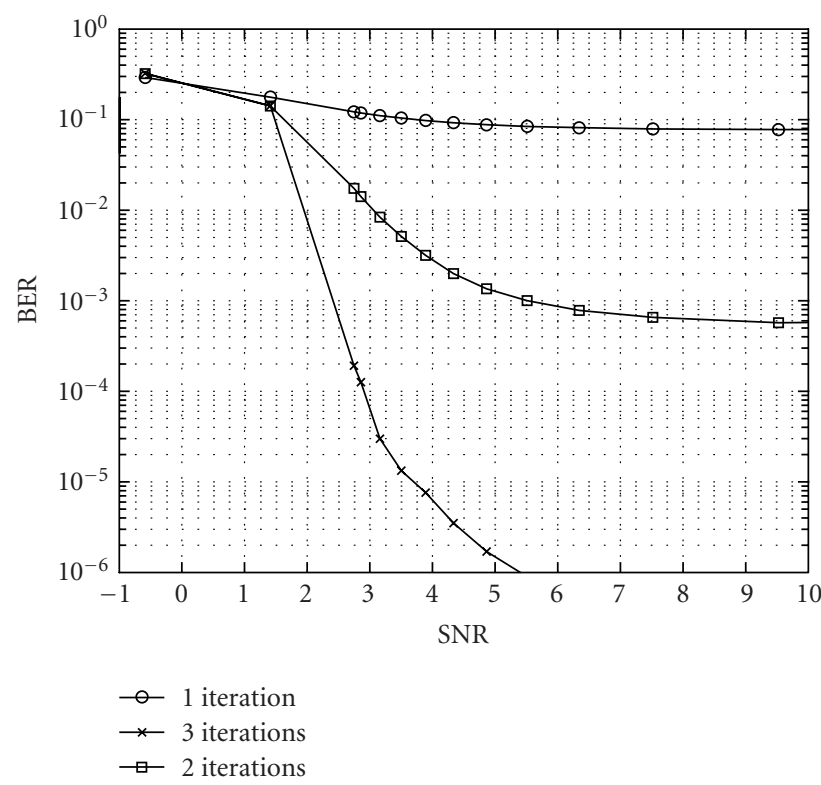

(a)

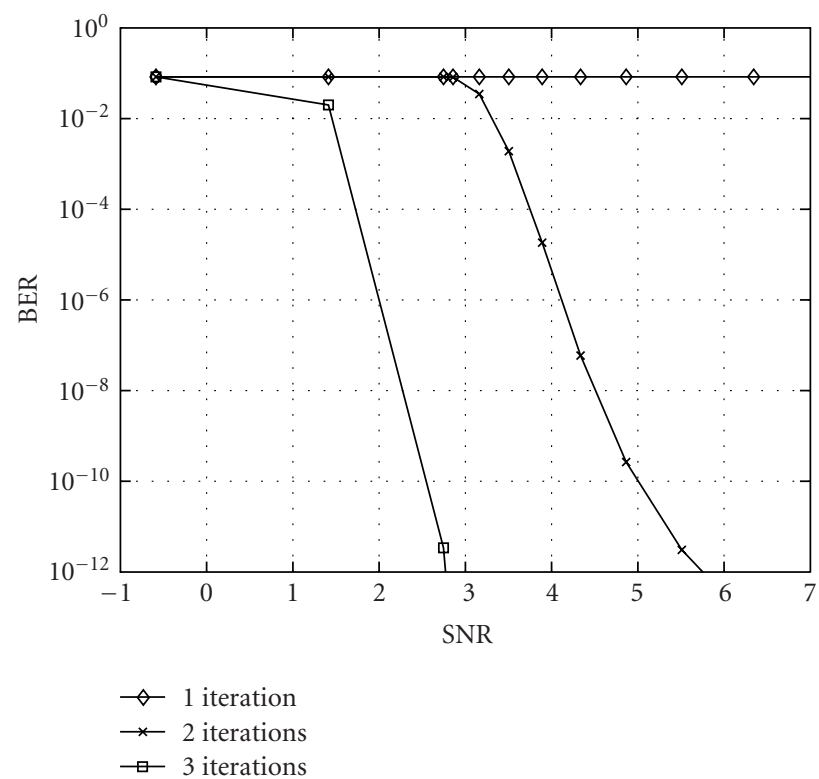

(b)

FIGURE 16: BER for a 67-user coded OCDMA transmission with a ternary spreading sequence of length 48: (a) after turbo decoding; (b) after turbo as well as RS decoding.

ratio (SNR) normalized to $E_{b} / N_{0}$. To increase the performance of the systems, we have constrained all the users' spreading sequences to have the same energy and to be separated from one another by at least a minimum Euclidean distance corresponding to 0.85 times their constant energy. In Figures 16 and 17, the BER is simulated using ternary and quaternary spreading sequences of length 48 , respectively. 


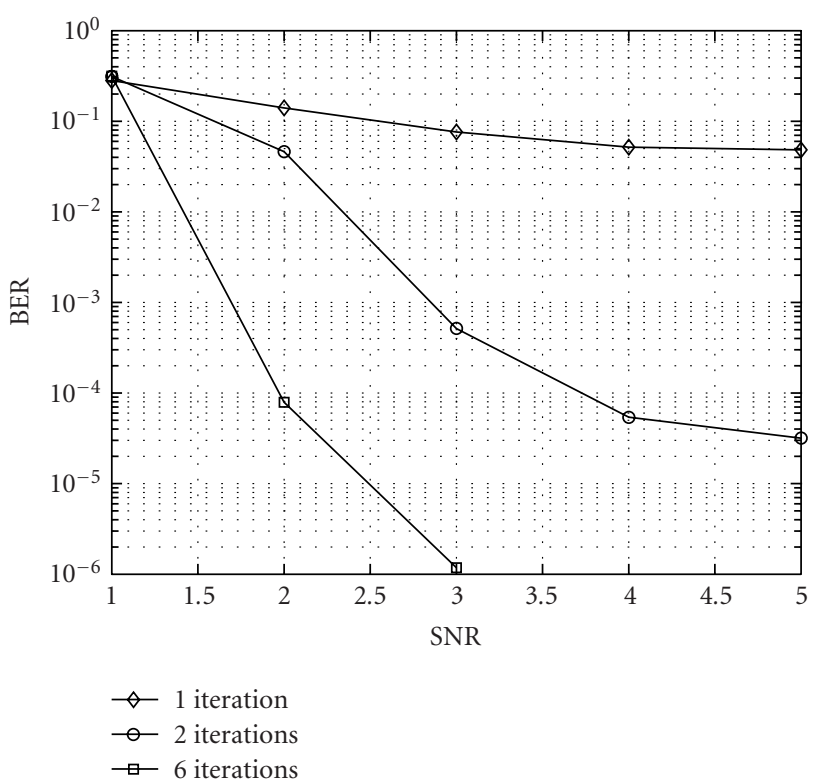

(a)

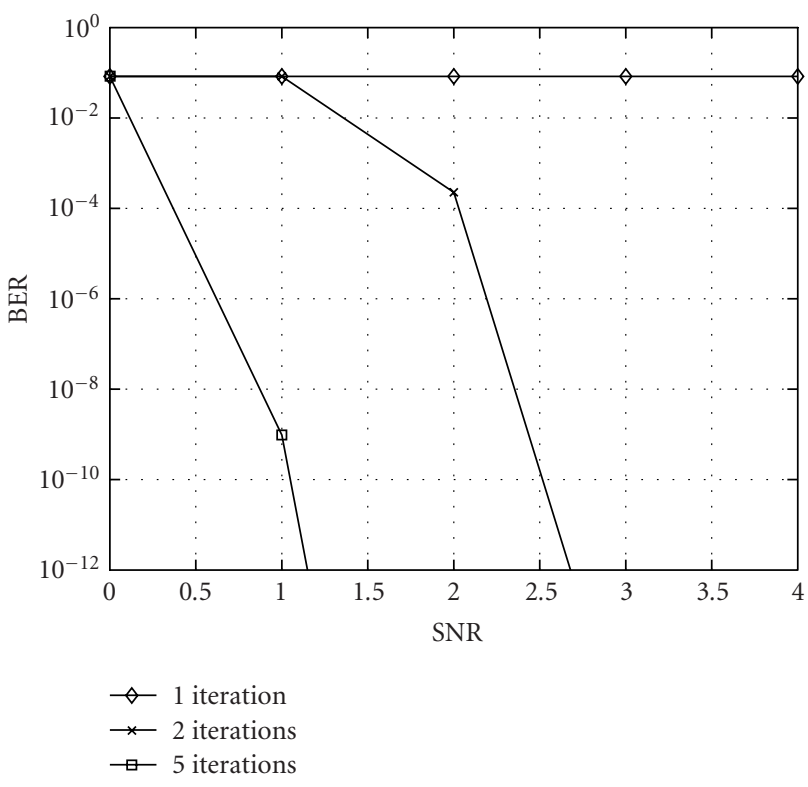

(b)

FIGURE 17: BER for a 57-user OCDMA transmission with a quaternary spreading sequence of length 48: (a) after turbo decoding; (b) after turbo as well as RS decoding.

It can be noticed that the transmission is overloaded in both cases with 67 and 57 active users and that the system still performs well at low SNRs. It is also interesting to note that these transmissions are particularly interference limited, since, at a certain point, an increasing SNR does not improve the performance. This occurs as the multi-user interference becomes much higher than the noise variance and causes the performance degradation.

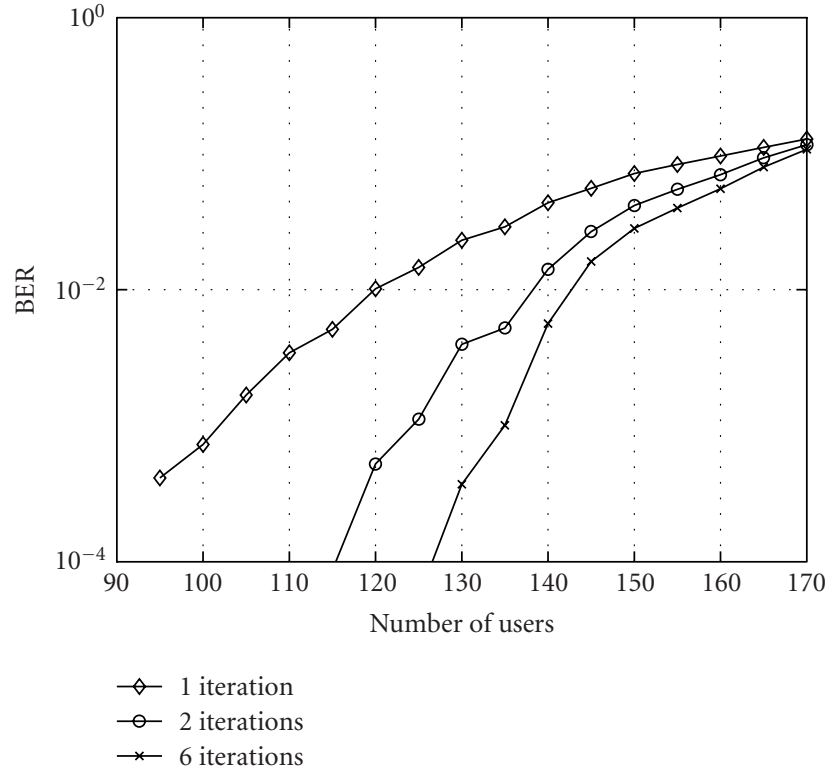

(a)

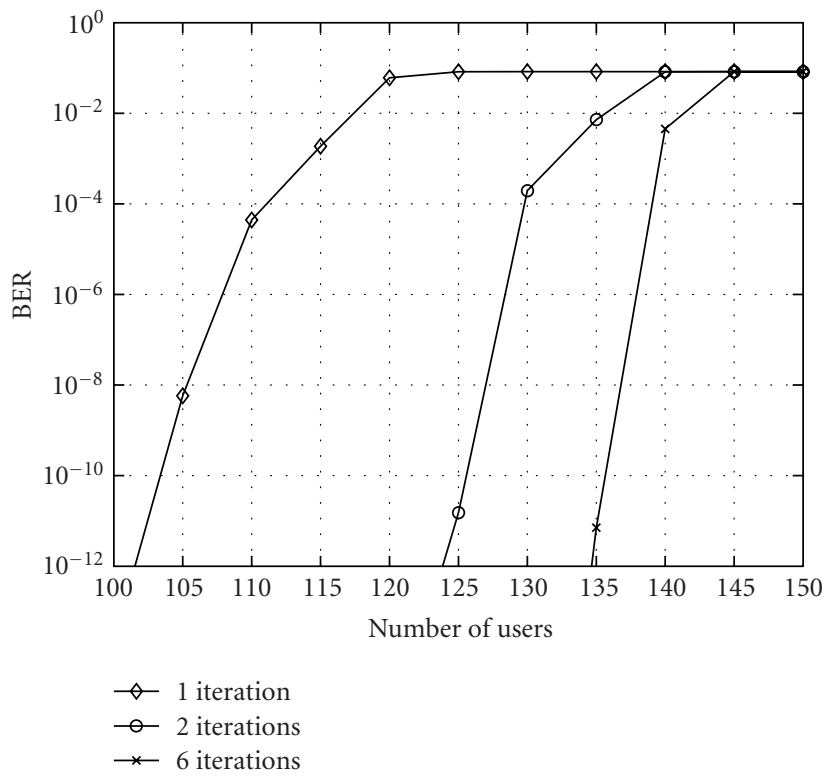

(b)

FIGURE 18: BER against the number of users for ternary OCDMA network with spreading sequences of length 128 and 10 dB SNR: (a) after turbo decoding; (b) after turbo as well as RS decoding.

In Figures 18 and 19, the simulated BER is plotted against the number of active OCDMA users for ternary and quaternary spreading sequences of length 128 and SNR $=10 \mathrm{~dB}$. In the case of quaternary modulation, for example, up to 150 total users (after 6 turbo iterations) can be supported with almost error-free transmission. It can be noted that the system is then overloaded since we have used spreading sequences with length 128 but still performs well for 150 users. 


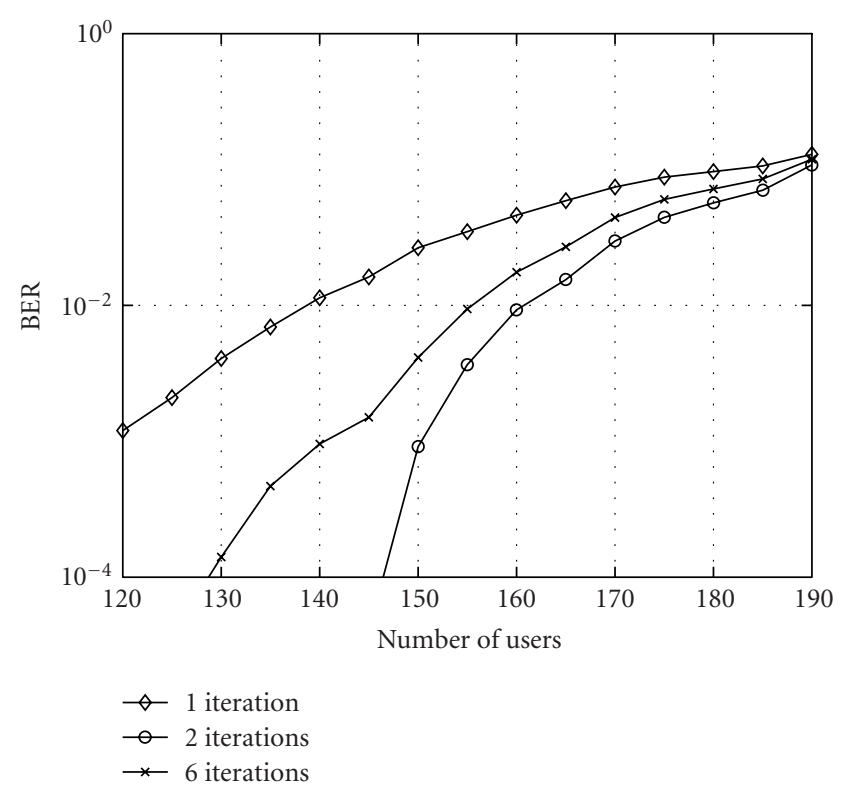

(a)

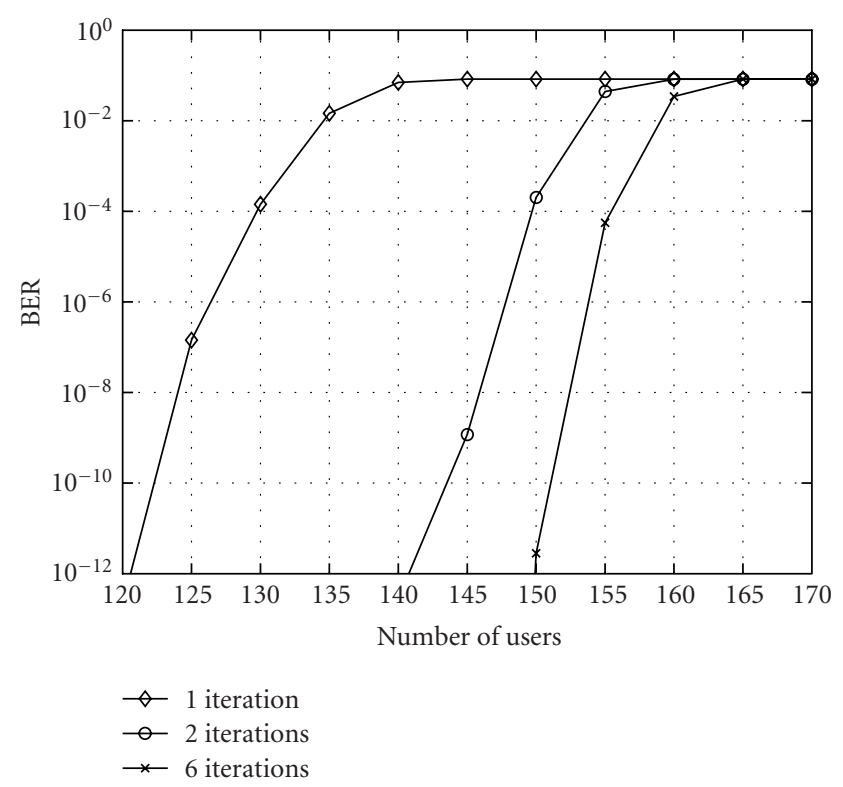

(b)

FIGURE 19: BER against the number of users for quaternary OCDMA network with spreading sequences of length 128 and $10 \mathrm{~dB}$ SNR: (a) after turbo decoding; (b) after turbo as well as RS decoding.

In addition, if the spreading sequences are constrained to have better cross-energy (higher minimum distance constraint), more users can be accommodated in the system with probability of error tending to zero.
It is important to note that in this part that the spreading sequences have been randomly generated and are hence not necessarily optimal. Therefore, the aggregate number of users can then be increased by using more elaborate spreading sequences. In our future work we will focus on the design of $M$-ary spreading sequences with better correlation properties for data recovery.

\section{CONCLUSION}

In this work, we have evaluated the channel capacity limits on optical CDMA transmission, when single-user detection is used at the receiver. We have shown that the theoretical network throughput of binary optical CDMA is limited by about 0.84 bits per OCDMA chip and that it can be improved by up to 4 times using $M$-level OCDMA spreading sequences. Hence, the $M$-ary modulation for OCDMA systems can be seen as one practical way to increase the capacity limitations of the binary optical CDMA.

In addition to the capacity calculations, we have also proposed and explored specific architectures for bitasynchronous OCDMA transmission. Using a concatenation of Berrou's turbo codes and Reed-Solomon codes, we have proposed a coded binary OCDMA system that can support several hundred active users on a multi-access network. Furthermore, we have also explored a turbo coded $M$ ary OCDMA transmission with single-user detection. Using proposed pseudorandomly generated spreading sequences based on near-capacity-achieving distributions, the $M$-ary OCDMA transmission schemes achieve good BER performance and support overloading at sufficiently low SNRs. The attractiveness of the proposed scheme lies in its reasonable complexity (e.g., due to the use of single-user detection), the utmost flexibility of the OCDMA network access scheme and ability to overload the system.

Our future work will focus on improving the proposed coding scheme to close the gap to the Shannon limit. We will also further explore a coding scheme for higher $M$ ary modulation levels and evaluate the optical CDMA system performance limits under different modes of channel noise.

\section{ACKNOWLEDGMENTS}

The authors would like to thank the anonymous reviewers for their constructive comments that helped to improve this manuscript. This research was supported by the Natural Sciences and Engineering Research Council (Canada), the Québec Fonds pour la Formation de Chercheurs et l'Aide à la Recherche, and the Canadian Foundation for Innovation. This work was presented in part at the Biennial Symposium on Communications, Kingston, Ontario, June 2002, the International Symposium on Turbo Codes, Brest, France, September 2003, and the IASTED International Multi-Conference on Wireless and Optical Communications, Banff, Alberta, July 2004. 


\section{REFERENCES}

[1] A. A. Shaar and P. A. Davies, "Prime sequences: quasi-optimal sequences for optical channel code division multiplexing," Electronics Letters, vol. 19, no. 21, pp. 888-889, 1983.

[2] P. Prucnal, M. Santoro, and T. Fan, "Spread spectrum fiberoptic local area network using optical processing," J. Lightwave Technol., vol. 4, no. 5, pp. 547-554, 1986.

[3] J. A. Salehi, "Code division multiple-access techniques in optical fiber networks. I. Fundamental principles," IEEE Trans. Commun., vol. 37, no. 8, pp. 824-833, 1989.

[4] R. M. Gagliardi and A. J. Mendez, "Pulse combining and timespace coding for multiple-accessing with fiber arrays," in Proc. IEEE/LEOS Summer Topical Meeting on Optical Multiple Access Networks, pp. 45-46, Monterey, Calif, USA, July 1990.

[5] L. Tančevski and I. Andonovic, "Wavelength hopping/time spreading code division multiple access systems," Electronics Letters, vol. 30, no. 17, pp. 1388-1390, 1994.

[6] A. J. Mendez and R. M. Gagliardi, "Code division multiple access (CDMA) enhancement of wavelength division multiplexing (WDM) systems," in Proc. IEEE International Conference on Communications (ICC '95), vol. 1, pp. 271-276, Seattle, Wash, USA, June 1995.

[7] M. Brandt-Pearce and B. Aazhang, "Performance analysis of single-user and multiuser detectors for optical code division multiple access communication systems," IEEE Trans. Commun., vol. 43, no. 234, pp. 435-444, 1995.

[8] K. Yu and N. Park, "Design of new family of two-dimensional wavelength-time spreading codes for optical code division multiple access networks," Electronics Letters, vol. 35, no. 10, pp. 830-831, 1999.

[9] H. M. H. Shalaby, "Chip-level detection in optical code division multiple access," J. Lightwave Technol., vol. 16, no. 6, pp. 1077-1087, 1998.

[10] H. Fathallah, L. A. Rusch, and S. LaRochelle, "Passive optical fast frequency-hop CDMA communications system," J. Lightwave Technol., vol. 17, no. 3, pp. 397-405, 1999.

[11] R. F. Ormondroyd and M. M. Mustapha, "Optically orthogonal CDMA system performance with optical amplifier and photodetector noise," IEEE Photon. Technol. Lett., vol. 11, no. 5, pp. 617-619, 1999.

[12] A. Stok and E. H. Sargent, "Lighting the local area: optical code-division multiple access and quality of service provisioning," IEEE Network, vol. 14, no. 6, pp. 42-46, 2000.

[13] S. Zahedi and J. A. Salehi, "Analytical comparison of various fiber-optic CDMA receiver structures," J. Lightwave Technol., vol. 18, no. 12, pp. 1718-1727, 2000.

[14] E. Inaty, H. M. H. Shalaby, P. Fortier, and L. A. Rusch, "Multirate optical fast frequency hopping CDMA System using power control," J. Lightwave Technol., vol. 20, no. 2, pp. 166176, 2002.

[15] R. M. H. Yim, L. R. Chen, and J. Bajcsy, "Design and performance of 2-D codes for wavelength-time optical CDMA," IEEE Photon. Technol. Lett., vol. 14, no. 5, pp. 714-716, 2002.

[16] A. J. Mendez and R. M. Gagliardi, "Combined effectiveness of optical hard-limiting and guard-time in optical CDMA systems," in Proc. IEEE Lasers and Electro-Optics Society (LEOS '02), vol. 2, pp. 857-858, Glasgow, UK, November 2002.

[17] R. M. H. Yim, J. Bajcsy, and L. R. Chen, "A new family of 2D wavelength-time codes for optical CDMA with differential detection," IEEE Photon. Technol. Lett., vol. 15, no. 1, pp. 165$167,2003$.

[18] A. J. Mendez, R. M. Gagliardi, V. J. Hernandez, C. V. Bennett, and W. J. Lennon, "Design and performance analysis of wave- length/time (W/T) matrix codes for optical CDMA," J. Lightwave Technol., vol. 21, no. 11, pp. 2524-2533, 2003.

[19] M. R. Dale and R. M. Gagliardi, "Channel coding for asynchronous fiberoptic CDMA communications," IEEE Trans. Commun., vol. 43, no. 9, pp. 2485-2492, 1995.

[20] J. Y. Kim and H. V. Poor, "Turbo-coded packet transmission for an optical CDMA network," J. Lightwave Technol., vol. 18, no. 12, pp. 1905-1915, 2000.

[21] P. Azmi, M. Nasiri-Kenari, and J. A. Salehi, "Soft-input decoder for decoding of internally channel coded fiber-optic CDMA communication systems," IEEE Trans. Commun., vol. 50, no. 12, pp. 1994-2002, 2002.

[22] R. M. H. Yim, J. Bajcsy, and L. R. Chen, "Optical CDMA transmission enabled by turbo codes," in Proc. International Symposium on Turbo Codes, pp. 571-574, Brest, France, September 2003.

[23] A. Mecozzi and M. Shtaif, "On the capacity of intensity modulated systems using optical amplifiers," IEEE Photon. Technol. Lett., vol. 13, no. 9, pp. 1029-1031, 2001.

[24] E. E. Narimanov and P. Mitra, "The channel capacity of a fiber optics communications system: perturbation theory," J. Lightwave Technol., vol. 20, no. 3, pp. 530-537, 2002.

[25] H. M. H. Shalaby, "Complexities, error probabilities, and capacities of optical OOK-CDMA communication systems," IEEE Trans. Commun., vol. 50, no. 12, pp. 2009-2017, 2002.

[26] R. M. H. Yim, J. Bajcsy, L. R. Chen, and C. Beainy, "On the capacity limit of asynchronous OCDMA with single user detection," in Proc. Biennial Symposium on Communications, pp. 485-489, Kingston, Ontario, Canada, June 2002.

[27] T. M. Cover and J. A. Thomas, Elements of Information Theory, John Wiley \& Sons, New York, NY, USA, 1991.

[28] S. Arimoto, "An algorithm for computing the capacity of arbitrary discrete memoryless channels," IEEE Trans. Inform. Theory, vol. 18, no. 1, pp. 14-20, 1972.

[29] S. B. Wicker and V. K. Bhargava, Eds., Reed-Solomon Codes and Their Applications, IEEE Press, Piscataway, NJ, USA, 1994.

[30] C. Berrou, A. Glavieux, and P. Thitimajshima, "Near shannon limit error-correcting coding and decoding: turbo-codes (1)," in Proc. IEEE International Conference on Communications (ICC '93), pp. 1064-1070, Geneva, Switzerland, May 1993.

Aminata A. Garba received a B.Eng. degree from Laval University in 2002 and an M.Eng. degree from McGill University in 2004, both in electrical engineering. She is presently a Ph.D. student at McGill University and her research interests include optical communications, information theory, and coding.

Raymond M. H. Yim received his B.Eng. and M.Eng. degrees in electrical engineering from McGill University in 2001 and 2002, respectively. He is currently pursuing his Ph.D. studies at Harvard University and his current research interests include switching and scheduling, high-speed access networks, and wireless communications.
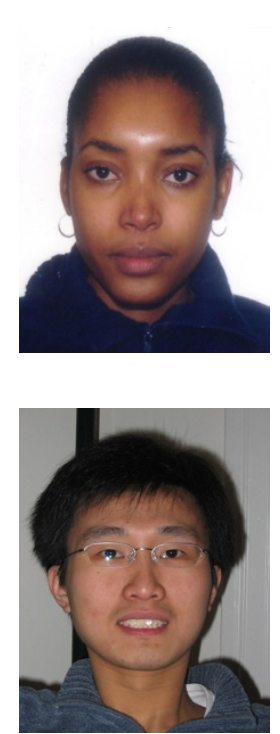
Jan Bajcsy received a B.S. degree in engineering sciences from Harvard University in 1994 and M.A. and Ph.D. degrees in electrical engineering from Princeton University in 1997 and 1999, respectively. He is presently an Assistant Professor of electrical engineering at McGill University and his research interests include optical and wireless communication systems, coding, and information theory.

Lawrence R. Chen received the B.Eng. degree in electrical engineering and mathematics from McGill University in 1995 and the M.A.S. and Ph.D. degrees in electrical and computer engineering from the University of Toronto in 1997 and 2000, respectively. He is presently an Assistant Professor in the Department of Electrical and Computer Engineering, McGill University, and his research interests focus on ultrafast pho-
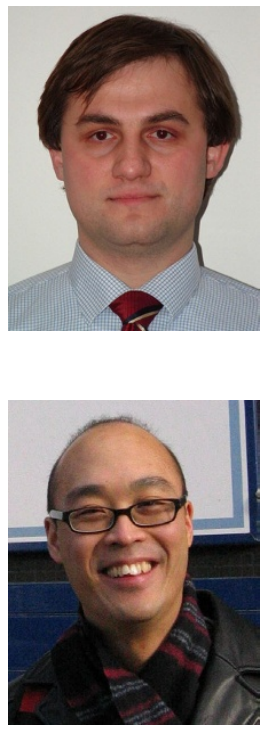

tonics, fiber optics, and optical communication systems. 